15

\title{
Нейтроноводная система ультрахолодных и холодных нейтронов на реакторе BBP-M
}

\author{
(C) А.П. Серебров, В.А. Лямкин, В.М. Пусенков, М.С. Онегин, А.К. Фомин, О.Ю. Самодуров, А.Т. Опрев, \\ В.А. Илатовский, Ю.Н. Журавлев, А.Ф. Щебетов, В.Г. Сыромятников, Г.П. Гордеев, Л.А. Аксельрод, \\ В.Н. Забенкин, И.В. Голосовский, О.П. Смирнов, В.Т. Лебедев, Ю.П. Черненков, В.В. Рунов
}

Петербургский институт ядерной фризики им. Б. П. Константинова, Национального исследовательского центра „Курчатовский Институт“, 188300 Гатчина, Россия

e-mail: serebrov@pnpi.spb.ru

Поступило в Редакцию 18 октября 2017 г. В окончательной редакции 20 ноября 2018 г. Принято к публикации 20 ноября 2018 г.

\begin{abstract}
Представлен расчет нейтронных потоков ультрахолодных (УХН), очень холодных и холодных нейтронов на выходах нейтроноводов источника УХН со сверхтекучим гелием на реакторе ВВР-М. В результате оптимизации параметров источника получена плотность УХН в ловушке спектрометра электрического дипольного момента (ЭДМ) $\rho_{351}=1.3 \cdot 10^{4} \mathrm{n} / \mathrm{cm}^{3}$. Полученная плотность УХН в ЭДМ-спектрометре на 2 порядка величины превышает плотность УХН на выходе существующих источников УХН. Плотность потока холодных нейтронов с длиной волны 2-20 $\AA$ на выходе нейтроновода с сечением $30 \times 200 \mathrm{~mm}^{2}$ составит $1.1 \cdot 10^{8} \mathrm{n} /\left(\mathrm{cm}^{2} \cdot \mathrm{s}\right)$. Плотность потока очень холодных нейтронов $(50-100 \AA)$ на выходе нейтроновода с сечением $30 \times 200 \mathrm{~mm}^{2}$ составит $2.3 \cdot 10^{5} \mathrm{n} /\left(\mathrm{cm}^{2} \cdot \mathrm{s}\right)$. На источнике запланирована обширная научная программа в области фундаментальной и прикладной физики.
\end{abstract}

DOI: 10.21883/JTF.2019.05.47486.2516

\section{Введение}

В настоящее время в НИЦ „Курчатовский институт“ - ПИЯФ разработан проект источника ультрахолодных нейтронов (УХН) для реактора ВВР-М [1]. Метод получения ультрахолодных нейтронов, используя сверхтекучий гелий, является очень перспективным Он основан на эффекте накопления нейтронов в сверхтекучем гелии из-за особенностей этой квантовой жидкости [2]. Уже проведен ряд успешных экспериментов в Японии и Франции на пучках холодных нейтронов. Проект будет весьма успешным шагом программы сохранения и развития фундаментальных и прикладных исследований в России на базе исследовательских реакторов. В источнике будет использоваться сверхтекучий гелий, который позволит достичь плотности ультрахолодных нейтронов $10^{3}-10^{4} \mathrm{n} / \mathrm{cm}^{3}$, которая еще нигде в мире не достигнута. Преодоление этого барьера послужит существенным прорывом в прогрессе фундаментальных и прикладных научных исследованиях.

Принцип получения УХН на сверхтекучем гелии подтвержден экспериментально, стоит лишь вопрос о практическом использовании этого эффекта и инженерном решении этой задачи. Сейчас источники УХН на основе сверхтекучего гелия преимущественно разрабатываются на выведенных нейтронных пучках в ряде научных центров Европы, Америки, Японии и Канады. На реакторе ВВР-М есть уникальная возможность существенно увеличить производительность источника УХН, разместив камеру источника в тепловой колоне реактора на расстоянии $30 \mathrm{~cm}$ от активной зоны. Такое расположение камеры источника позволит на 2 порядка увеличить плотность потока УХН в экспериментальных установках по сравнению с источниками на выведенных пучках. Однако при таком расположении источника УХН необходимо решить сложную инженерную задачу отведения $30 \mathrm{~W}$ теплопритоков от сверхтекучего гелия при температуре $1.2 \mathrm{~K}$, что было успешно сделано на полномасштабной модели источника УХН [3].

Гелий эффективно конвертирует нейтроны с длиной волны $9 \AA$ в ультрахолодные нейтроны, однако та часть холодных нейтронов, которые пройдут через гелий без конвертации в ультрахолодное состояние, будет выводиться в качестве пучков холодных и очень холодных нейтронов. Для эффективной работы источника в производстве ультрахолодных нейтронов он должен находиться в интенсивном потоке нейтронов с длиной волны $9 \AA$. Поэтому в носовую часть источника УХН необходимо поместить дополнительную камеру с предзамедлителем.

Кроме того, камера предзамедлителя может быть эффективным источником холодных нейтронов во всем диапазоне длин волн нейтронов больше $4 \AA$, которые будут выводиться из тепловой колонны и эффективно использоваться. В качестве рабочего тела предзамедлителя выбран жидкий дейтерий при температуре $20 \mathrm{~K}$, стандартно использующийся в источниках холодных и ультрахолодных нейтронов. Подробный анализ выбора предзамедлителя для источника УХН приведен в статье [4]. 


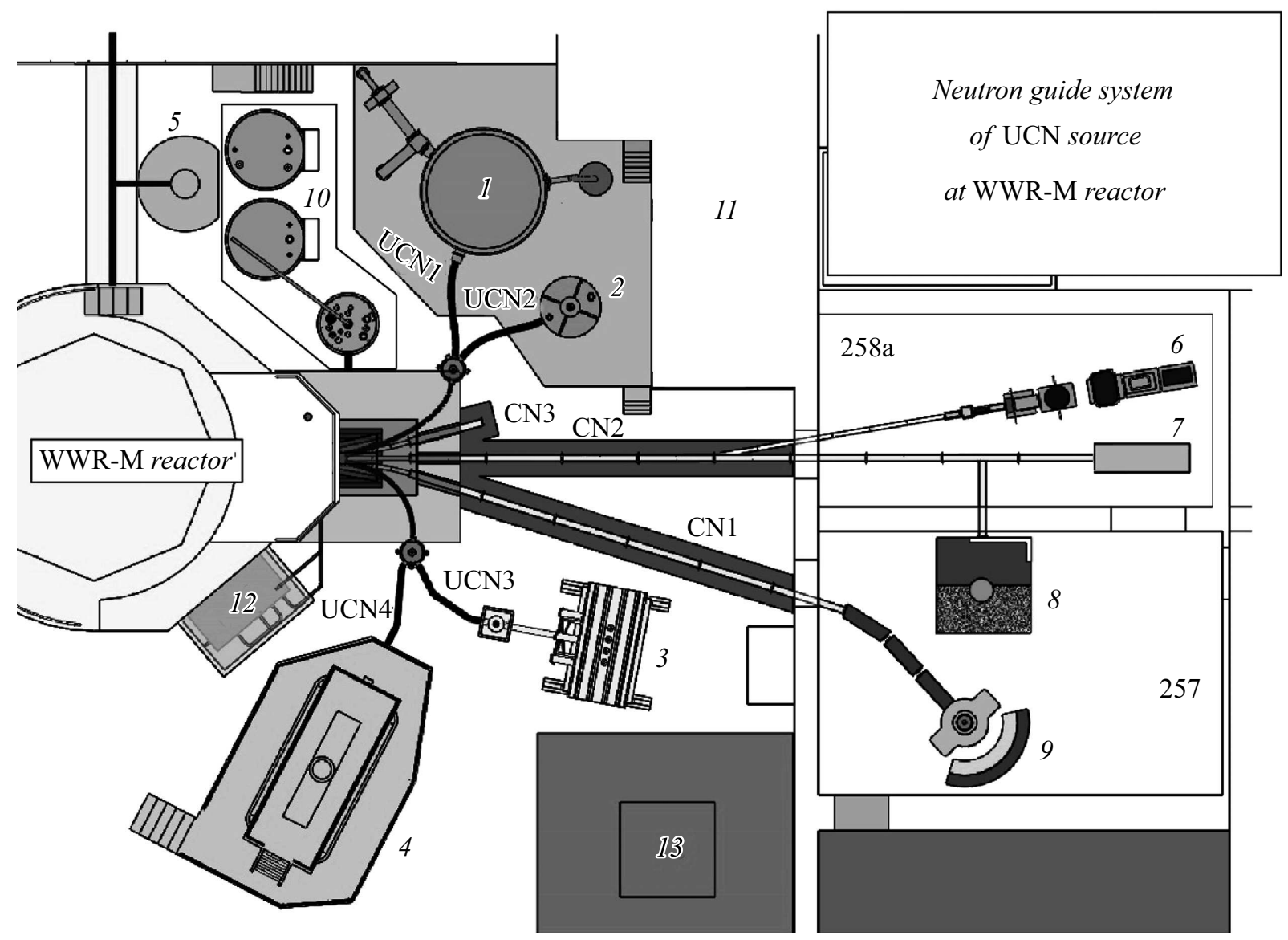

Рис. 1. Схема размещения экспериментального оборудования в главном зале реактора ВВР-М УХН-пучки ультрахолодных нейтронов, ХН-пучки холодных и очень холодных нейтронов: 1 - ЭДМ спектрометр, 2 - магнитная ловушка УХН, 3 эксперимент $n-n^{\prime}, 4-$ гравитационная ловушка УХН, 5 - дифрактометр, 6 - рефлектометр, 7 - поляриметр, 8 - порошковый дифрактометр, 9 - спин-эхо спектрометр, 10 - криогенное оборудование источника УХН, 11 - технологическая платформа для экспериментального оборудования, 12 - система охлаждения свинцового экрана источника УХН, 13 - транспортный въезд в главный зал реактора ВВР-М.

Постановка задачи. Для нового источника низкоэнергетических нейтронов требуется спроектировать и рассчитать нейтроноводную систему. Данную систему необходимо вписать в текущую геометрию главного зала реакторного комплекса ВВР-М и оптимизировать для экспериментального оборудования, которое будет использовать пучки УХН, очень холодных нейтронов $(\mathrm{OXH})$ и холодных нейтронов (ХН). При проектировании нейтроноводов необходимо отсечь весь спектр гамма квантов и быстрых нейтронов идущих напрямую из реактора отклонением нейтроноводов на некоторый угол.

\section{1. Расположение нейтроноводной системы холодных и очень холодных нейтронов в главном зале реактора ВBP-M}

Источник ультрахолодных нейтронов располагается в северной части главного зала реакторного комплекса
ВВР-М. На рис. 1 показана схема размещения экспериментальных установок для работы с источником УХН.

С использованием нового источника УХН предполагается улучшить точность измерений электрического дипольного момента (ЭДМ) нейтрона 1 на два порядка величины и проверить предсказания суперсимметричных теорий, которые являются одним из вариантов расширения Стандартной Модели. В рамках этих теорий ЭДМ нейтрона предсказывается на уровне, доступном для планируемых экспериментов. В то же время суперсимметричные теории предсказывают барионную асимметрию Вселенной на наблюдаемом уровне, что указывает на возможную справедливость предложенных вариантов теории.

Помимо установки для измерения ЭДМ-нейтрона представлены две установки для измерения времени жизни нейтрона: с магнитной ловушкой 2 и с большой гравитационной ловушкой 4. Прецизионные измерения времени жизни нейтрона важны для проверки модели формирования Вселенной на ее ранней стадии, а также 


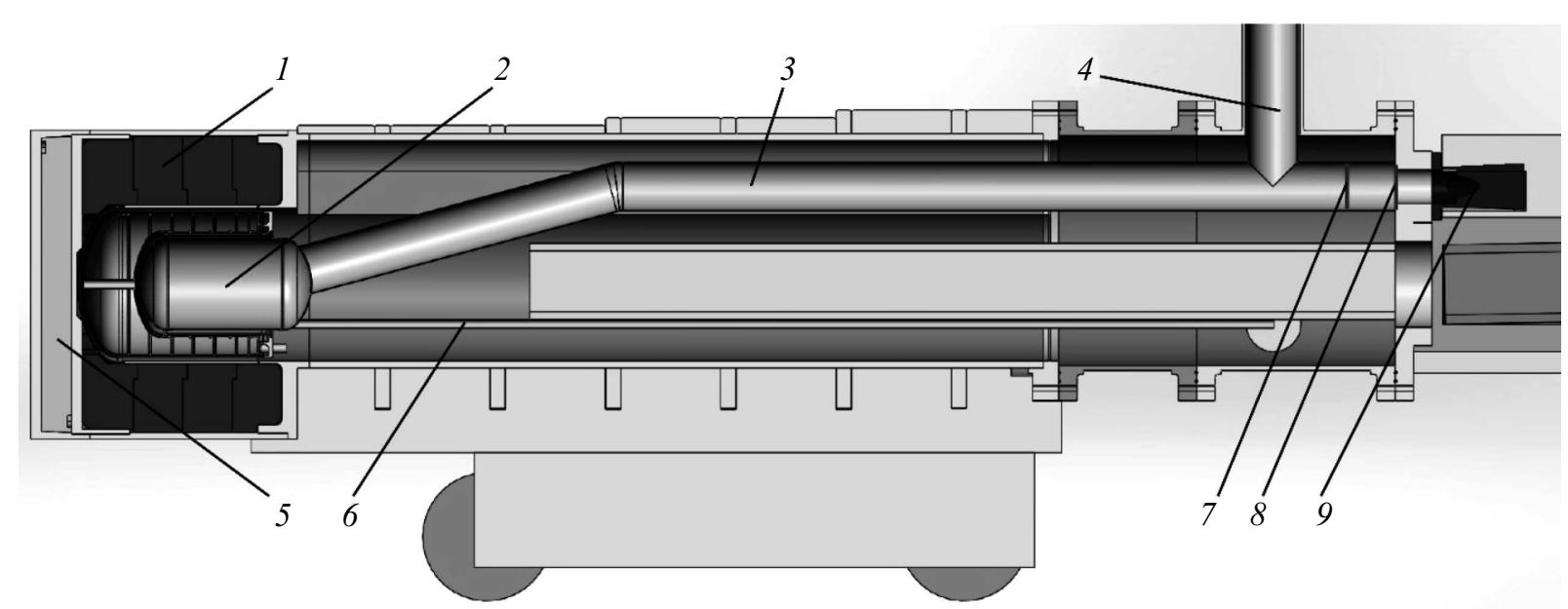

Рис. 2. Нейтроноводная система УХН источника УХН на реакторе ВВР-М. 1 - графит, 2 - источник УХН, ХН и ОХН, 3 нейтроновод УХН, 4 - откачка паров гелия, 5 - свинцовый экран, 6 - трубка подачи сверхтекучего гелия, 7 - холодная мембрана УХН, 8 - теплая мембрана УХН, 9 - разветвитель пучка УХН.

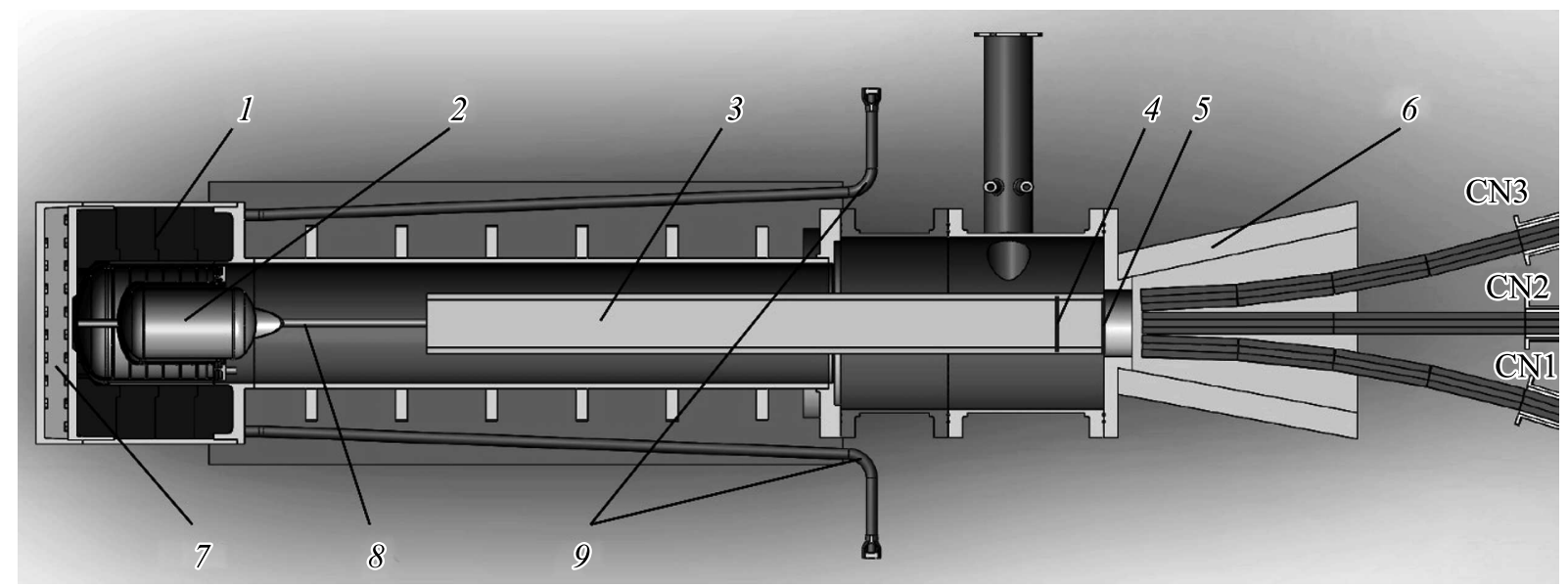

Рис. 3. Нейтроноводная система ХН и ОХН источника УХН на реакторе ВВР-М. 1 - графит, 2 - источник УХН, ХН и ОХН, 3 - нейтроновод ХН и ОХН, 4 - холодная мембрана ХН и ОХН, 5 - теплая мембрана ХН и ОХН, 6 - блок коллиматоров, 7 - свинцовый экран, 8 - трубка подачи сверхтекучего гелия, 9 - система охлаждения свинцового экрана.

для поиска отклонений от Стандартной модели. Кроме того, представлена установка для поиска зеркальной темной материи $\left(n-n^{\prime}\right)$ 3. Все эти установки разработаны и изготовлены в ПИЯФ и в настоящее время проходят испытания на пучках УХН в Институте ЛауэЛанжевена (ИЛЛ). Предполагается, что они будут перенесены в ПИЯФ на новый источник УХН. Увеличение интенсивности УХН более чем на два порядка величины позволит проводить принципиально новые исследования. Наконец, для высокоинтенсивного источника УХН можно обсуждать постановку эксперимента по поиску нейтрон-антинейтронных осцилляций $(n-\tilde{n})$ с целью проверки нарушения барионного числа, второго условия возникновения Вселенной по теореме А.Д. Сахарова. Таким образом, кроме важнейшего эксперимента по поиску ЭДМ нейтрона открываются возможности для проведения целой серии экспериментов по физике фундаментальных взаимодействий.

Программа исследований конденсированного состояния на пучках холодных нейтронов рассчитана на 5 экспериментальных станций. Среди них 4 готовых установки: Рефлектометр 6, поляриметр 7, порошковый дифрактометр 8 и спин-эхо спектрометр 9. Также предусмотрен вывод резервного пучка очень холодных нейтронов (OXH) (CN3) для будущих экспериментов.

Северная часть главного зала в большей части использована под криогенное оборудование источника УХН и экспериментальные станции на ультрахолодных нейтронов. Таким образом, установки на ХН и ОХН будут расположены в помещениях за пределами главного зала реакторного комплекса ВВР-М. Уровень пола в этих помещениях выше уровня пола в главном 
зале на $750 \mathrm{~mm}$. При высоте пучка холодных нейтронов в $1000 \mathrm{~mm}$ относительно уровня пола главного зала необходимо поднять пучок на $200 \mathrm{~mm}$ в конечной точке для возможности установки установок спин-эхо спектрометра, поляриметра и рефлектометра. Помимо всего прочего пучок, идущий на спин-эхо спектрометр, должен обойти технологическое отверстие для допуска в горячие камеры. Для этого нейтроновод для спинэхо нужно отклонить минимум на $17^{\circ}$ относительно исходного пучка.

При проектировании нейтроноводов необходимо отсечь весь спектр гамма квантов и быстрых нейтронов, идущих напрямую из реактора отклонением нейтроноводов на некоторый угол.

Существенной проблемой является то, что в нейтроноводах образуется существенный радиационный фон в помещении главного зала реактора ВВР-М. В связи с этим все нейтроноводы необходимо снабдить достаточной биологической защитой для создания приемлемой

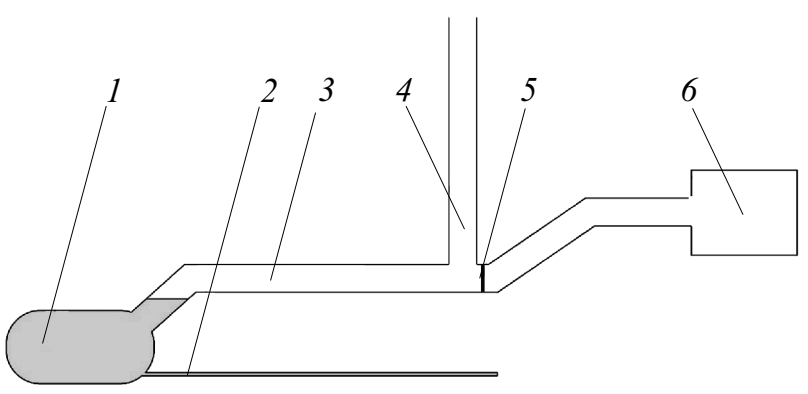

Рис. 4. Расчетная схема источника УХН: 1 - камера источника, 2 - трубка заливки камеры, 3 - нейтроновод УХН, 4 - трубопровод вакуумной откачки камеры (гравитационный затвор УХН), 5 - мембрана на выходе из камеры источника, 6 - ловушка УХН.

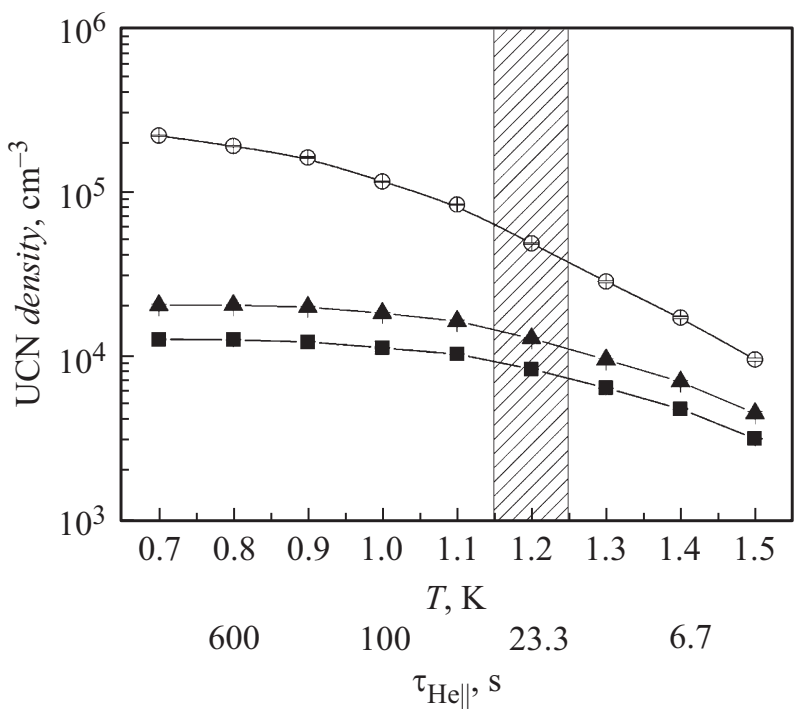

Рис. 5. Плотность УХН в зависимости от температуры НеІІ в камере источника: $\bullet$ - в закрытой камере источника, $\boldsymbol{\Delta}-$ в ловушке объемом $351, \mathbf{\square}-$ в ловушке объемом 3501.

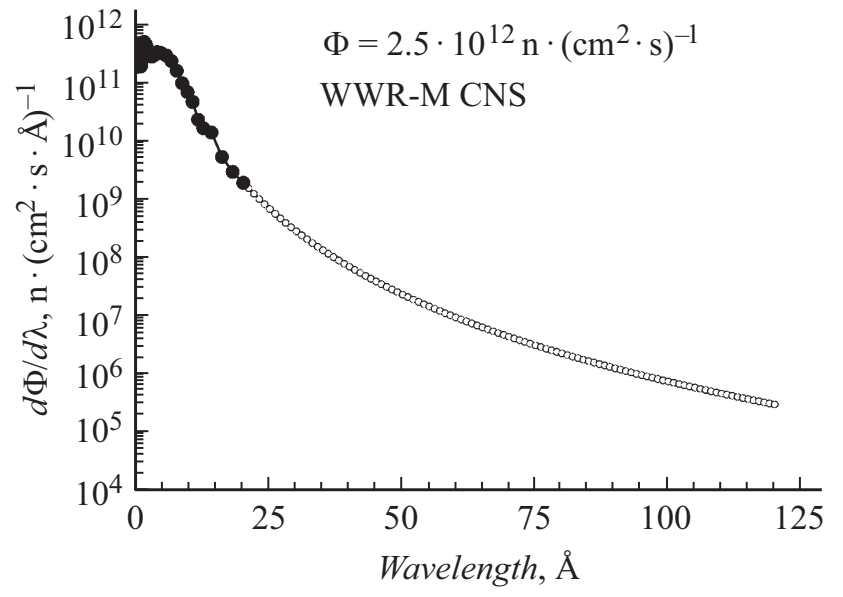

Pис. 6. Плотность нейтронного потока $d \Phi / d \lambda$ на поверхности источника холодных нейтронов.

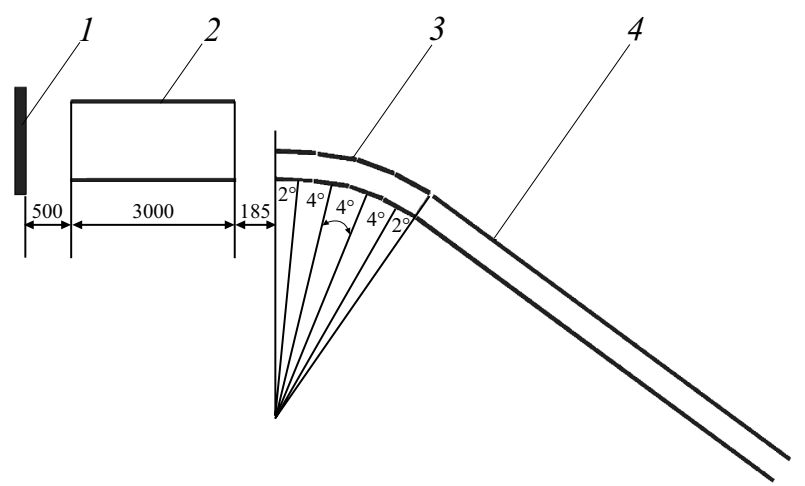

Рис. 7. Схема расположения источника холодных нейтронов и нейтроновода CN1. 1 - источник ИХН, 2 - нейтроновод, 3 - изогнутый нейтроновод, 4 - прямой нейтроновод.

радиационной обстановки обслуживающему инженерному и научному персоналу, работающим на экспериментальных станциях.

На основе разработанной схемы, была спроектирована нейтроноводная система ультрахолодных (рис. 2) холодных и очень холодных нейтронов (рис. 3).

\section{2. Нейтроноводная система ультрахолодных нейтронов}

Расчет и оптимизация выхода ультрахолодных нейтронов из источника проводились с помощью метода Монте-Карло с использованием программы [5], разработанной для нейтронных расчетов с учетом гравитации. В процессе моделирования варировались различные параметры с целью оптимизации выхода УХН.

Расчетная схема низкотемпературной части источника УХН приведена на рис. 4. Камера источника 1 имеет диаметр $30 \mathrm{~cm}$ и объем 351 . Сверхтекучий гелий поступает в камеру источника по трубке 2. Откачка НеII производится через трубопровод вакуумной откачки диа- 


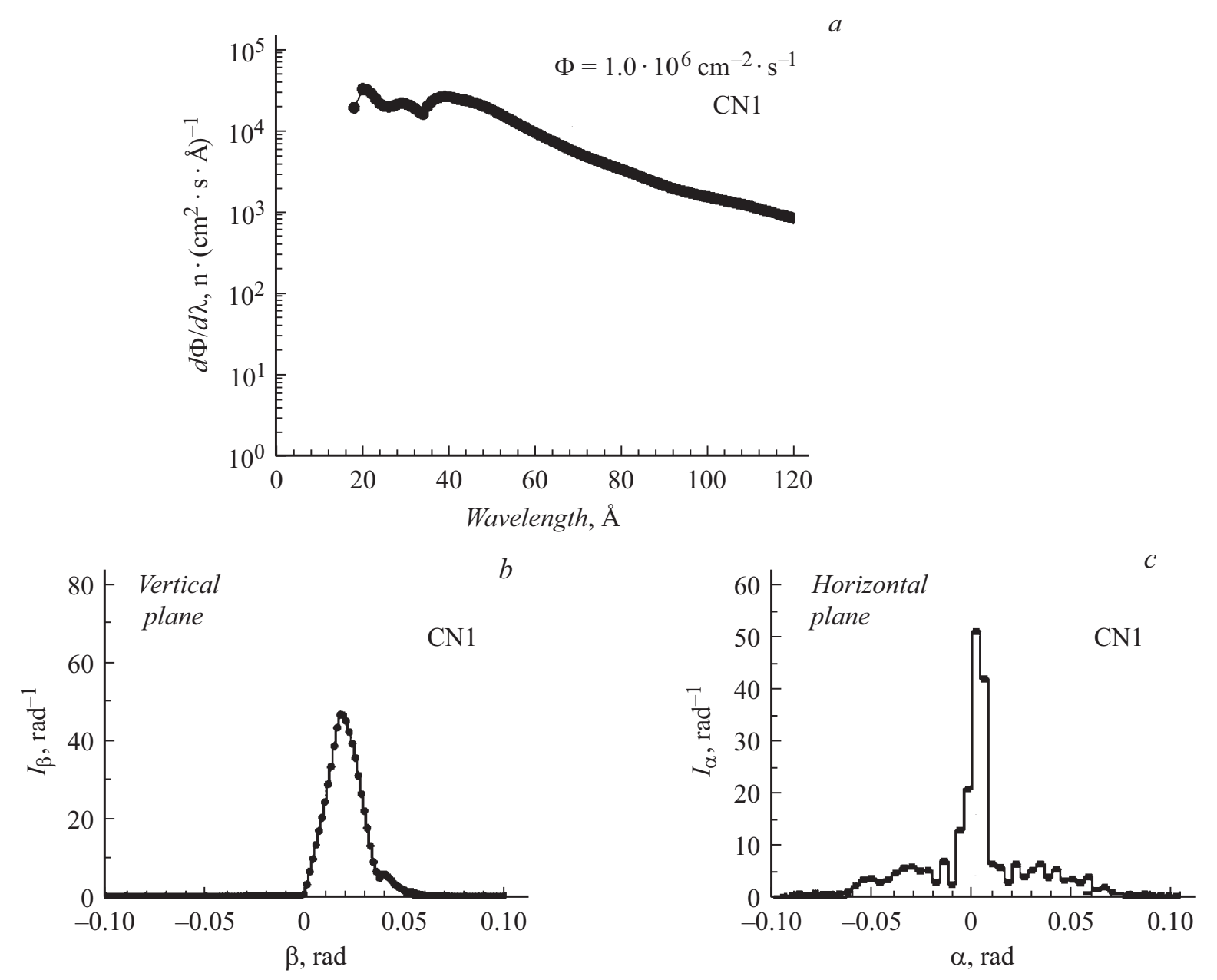

Рис. 8. $a-$ плотность нейтронного потока $d \Phi / d \lambda$ (сверху) на выходе нейтроновода CN1 длиной $7500 \mathrm{~mm}$ и сечением $30 \times 200 \mathrm{~mm}^{2}, b-$ угловое распределение интенсивности нейтронов $I_{\beta}, c-$ угловое распределение интенсивности нейтронов $I_{\alpha}$.

метром $100 \mathrm{~mm}$ 4. Такая схема откачки практически не создает утечку УХН, так как вертикальный трубопровод откачки создает гравитационный затвор для УХН. Совмещение трубопровода откачки и нейтроновода УХН является возможным, так как время хранения нейтронов в парах гелия при $T=15 \mathrm{~K}$ и $P=50 \mathrm{~Pa}$ составит $52 \mathrm{~s}$, что много больше чем время транспортировки по нейтроноводу.

Камера соединяется с ловушкой УХН 6 при помощи нейтроновода 3. Камера, нейтроноводы и ловушка УХН имеют внутри напыление $58 \mathrm{NiMo} \mathrm{c} \mathrm{граничной}$ скоростью $7.8 \mathrm{~m} / \mathrm{s}$ и коэффициентом потерь $3 \cdot 10^{4}$. При отражении нейтронов от стенок нейтроноводов $0.7 \%$ отражений являются диффузными, а при отражении от стенок камеры источника и ловушки УХН - 90\%.

Скорость производства УХН в НеII, содержащимся в источнике, составляет $(3.6-5) \cdot 10^{3} \mathrm{n} /\left(\mathrm{cm}^{3} \cdot \mathrm{s}\right)$. Однако для консервативной оценки мы выбираем скорость производства УХН равной $3 \cdot 10^{3} \mathrm{n} /\left(\mathrm{cm}^{3} \cdot \mathrm{s}\right)$.

В результате моделирования получена плотность УХН для ловушек объемом 35 и 3501 . Выбранные размеры ловушек характерны для экспериментов по измерению
ЭДМ нейтрона (351) [6] и времени жизни нейтрона (3501) [7]. На рис. 5 показана плотность УХН в зависимости от температуры HeII в камере источника. Вдоль оси абсцисс дополнительно отложены значения времени хранения нейтронов в HeII при данной температуре. Для сравнения на рис. 5 также показана плотность УХН в условно закрытой камере источника, чтобы представить какую плотность можно было бы достигнуть в режиме накопления. Однако мы работаем в режиме стационарного производства УХН в источнике, при этом достижимая плотность в экспериментальной ловушке объемом 351 в этом режиме падает приблизительно в 4 раза и оказывается еще на $54 \%$ меньше для ловушки на порядок большего объема. Эта плотность фактически является плотностью УХН в режиме вытекания на выходе открытого нейтроновода. Как видно из графика, эта плотность падает с ростом температуры, так как падает время хранения нейтронов. Расчет выполнен при диаметре нейтроновода $100 \mathrm{~mm}$, диаметре трубопровода вакуумной откачки $70 \mathrm{~mm}$, диаметре трубы заливки камеры $10 \mathrm{~mm}$. Установлены две алюминиевых мембраны: одна в низкотемпературной части нейтроновода, вторая 


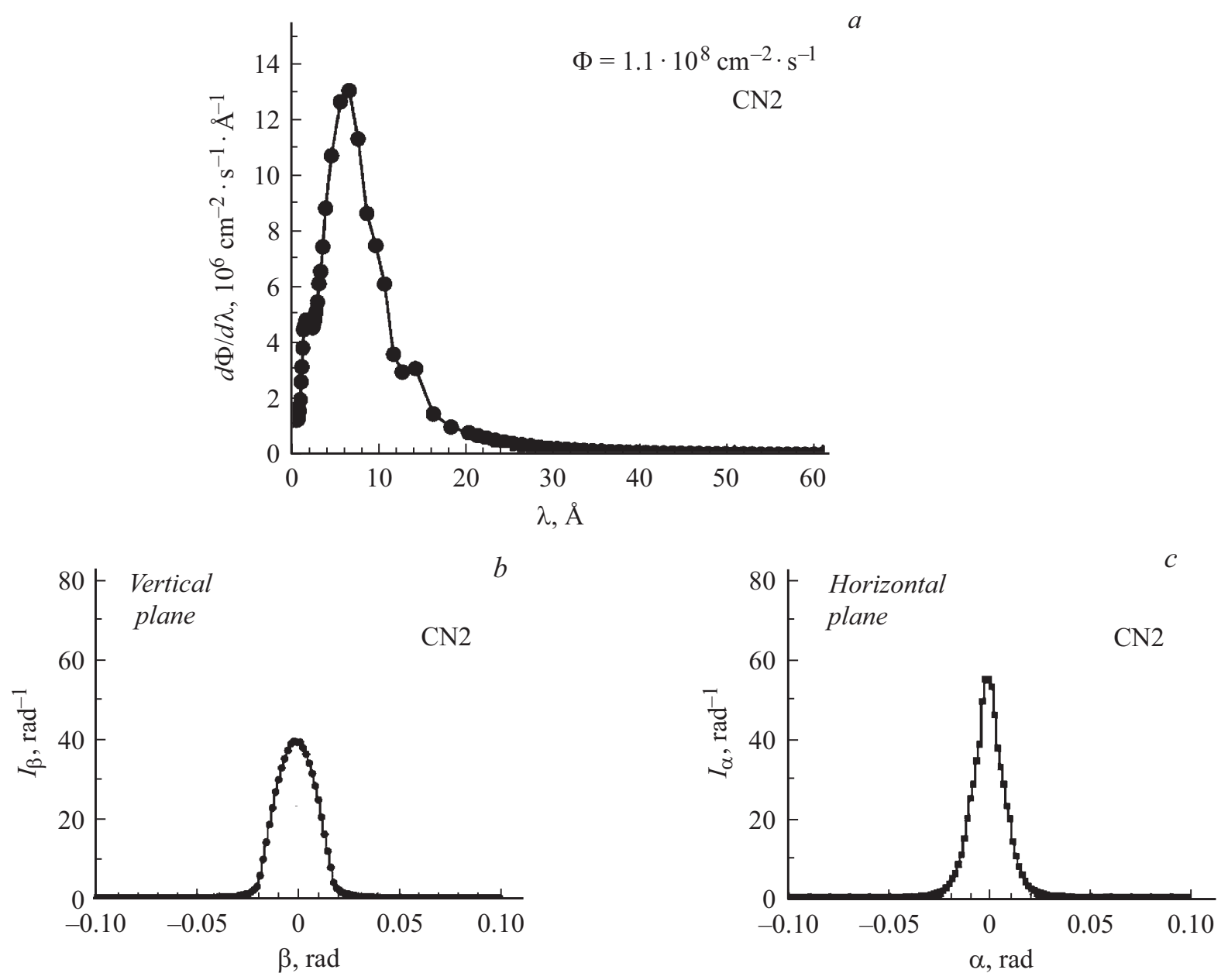

Рис. 9. $a-$ плотность нейтронного потока $d \Phi / d \lambda$ на выходе прямого нейтроновода CN2 длиной $7500 \mathrm{~mm}$ и сечением $30 \times 200 \mathrm{~mm}^{2}, b-$ угловое распределение интенсивности нейтронов $I_{\beta}, c-$ угловое распределение интенсивности нейтронов $I_{\alpha}$.

при комнатной температуре на выходе в разветвитель пучка УХН.

Таким образом, в результате оптимизации параметров источника получена плотность УХН в основной ловушке $\rho_{351}=1.3 \cdot 10^{4} \mathrm{n} / \mathrm{cm}^{3}$ (для ловушки объемом 351) и $\rho_{3501}=8.4 \cdot 10^{3} \mathrm{n} / \mathrm{cm}^{3}$ (для ловушки объемом 3501 ).

Ожидаемая плотность УХН на 2-3 порядка величины превысит плотность существующих источников УХН [8].

\section{3. Нейтроноводная система холодных и очень холодных нейтронов}

Плотность нейтронного потока $d \Phi / d \lambda$ на поверхности источника холодных нейтронов представлена на рис. 6. Для длин волн от 0 до $20 \AA$ (черные кружки) плотность нейтронного потока рассчитывалась методом Монте-Карло. Для длин волн от 20 до $120 \AA$ (белые кружки) плотность потока аппроксимировалась максвелловским распределением с температурой предзамедлителя $15 \mathrm{~K}$. Размер источника ХН имеет диаметр $320 \mathrm{~mm}$. Расстояние „источник ХН-нейтроновод“ равно
$500 \mathrm{~mm}$. Поток нейтронов на поверхности источника равен $2.5 \cdot 10^{12} \mathrm{~cm}^{-2} \cdot \mathrm{s}^{-1}$.

После источника УХН поток холодных нейтронов проходит через нейтроновод сечением $200 \times 200 \mathrm{~mm}^{2}$ с покрытием 58NiMo и попадает на „холодную“ и теплую алюминиевые мембраны с суммарной толщиной $0.76 \mathrm{~mm}$. Холодная мембрана исключает прямой просвет от вакуумного кожуха источника УХН $(T=300 \mathrm{~K})$ на низкотемпературный гелиевый источник $(T=1.2 \mathrm{~K})$. Теплая мембрана разделяет вакуумный объем внешних нейтроноводных систем от вакуумного объема источника. Далее пучок ХН разделяется на 3 пучка, идущих на экспериментальные установки. Условно эти пучки имеют обозначение: CN1 (спин-эхо спектрометр), CN2 (порошковый дифрактометр, поляриметр, рефлектометр) и CN3 (резерв).

Нейтроноводы изготавливаются из оптического стекла К8 (ГОСТ 3514-94). Для улучшения свойств нейтроноводной системы внутренняя поверхность нейтроновода напылена изотопом $58 \mathrm{Ni}$ с граничной скоростью отражения $8 \mathrm{~m} / \mathrm{s}$. Сечение прямого нейтроновода имеет 

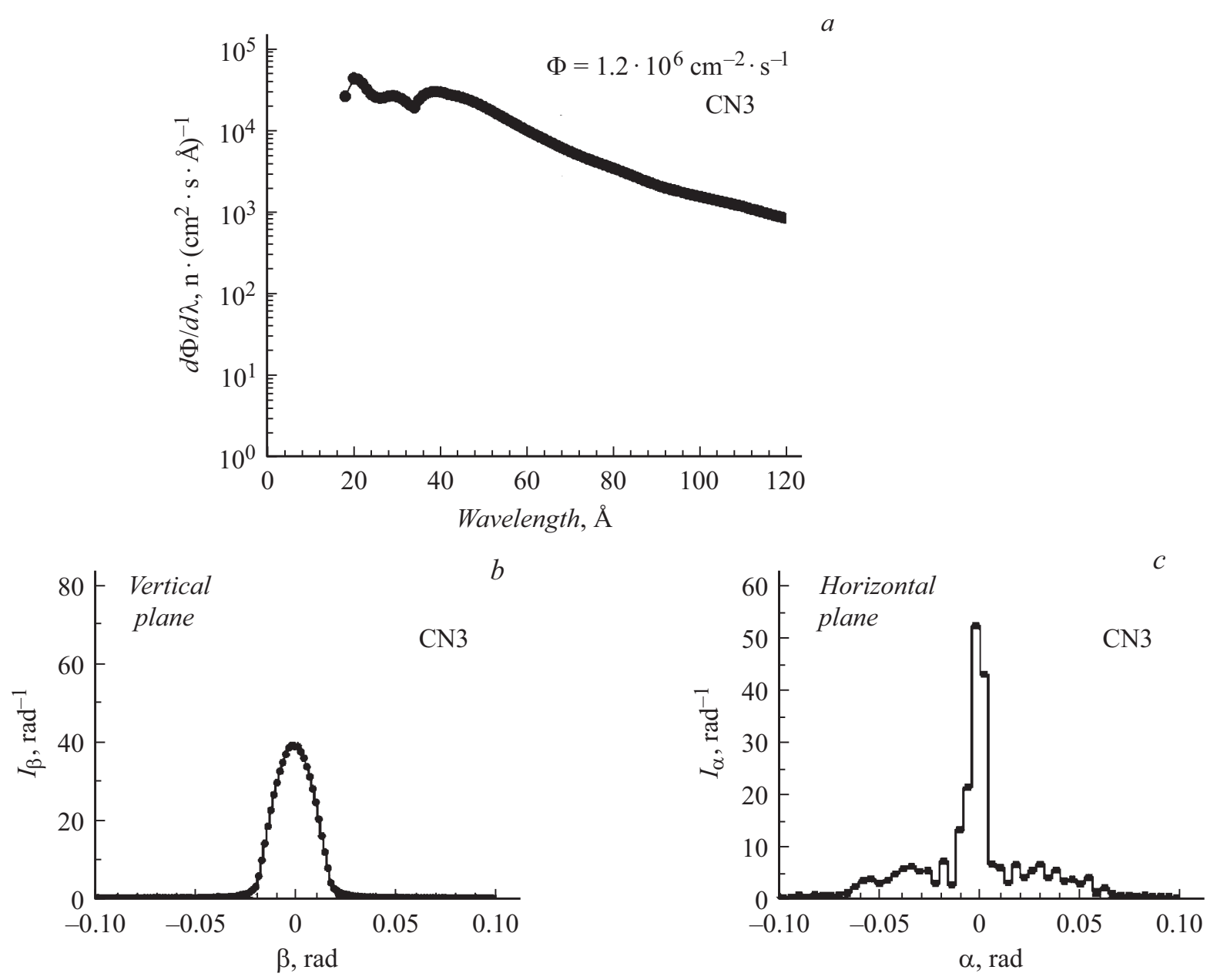

Рис. 10. $a-$ плотность нейтронного потока $d \Phi / d \lambda$ (сверху) на выходе нейтроновода CN3 длиной $7500 \mathrm{~mm}$ и сечением $30 \times 200 \mathrm{~mm}^{2}, b-$ угловое распределение интенсивности нейтронов $I_{\beta}, c-$ угловое распределение интенсивности нейтронов $I_{\alpha}$.

размеры $30 \times 200 \mathrm{~mm}^{2}$ при длине секции нейтроновода в $800 \mathrm{~mm}$.

Основные элементы нейтроноводной системы изготовлены. Материал вакуумного кожуха нейтроноводов - сплав АМг. На всей длине нейтроноводы окружены защитой от гамма и нейтронного излучения. Нейтроноводы откачиваются безмасляным форвакуумным насосом.

\section{1. Нейтроновод $\mathrm{CN} 1$}

Общая схема размещения источника холодных нейтронов и нейтроновода $\mathrm{CN} 1$ представлена на рис. 7. Изогнутая часть нейтроновода длиной $1600 \mathrm{~mm}$, сечением $30 \times 200 \mathrm{~mm}^{2}$ состоит из четырех секций длиной $400 \mathrm{~mm}$. Каждая секция отклоняется от предыдущей на угол 2 или $4^{\circ}$ как указано на рис. 7. Общее отклонение выведенного пучка относительно исходного составит $16^{\circ}$. Этот изгиб необходим для обхода технологического люка обслуживания горячих камер в полу главного зала реактора ВВР-М. Помимо этого нейтроновод CN1 необходимо поднять на $200 \mathrm{~mm}$ в
Таблица 1. Параметры нейтронно-оптических элементов нейтроноводов ХН и ОХН

\begin{tabular}{l|c|c}
\hline \multicolumn{1}{c|}{ Название элемента } & Сечение, $\mathrm{mm}^{2}$ & Длина, $\mathrm{mm}$ \\
\hline Нейтроновод & $200 \times 200$ & 3000 \\
Коллиматор блока & $200 \times 200$ & 800 \\
Прямой нейтроновод $(\mathrm{CN} 1$ и $\mathrm{CN} 2)$ & $30 \times 200$ & 4300 \\
Прямой нейтроновод $(\mathrm{CN} 3)$ & $30 \times 200$ & 2400
\end{tabular}

конечной точке из-за разницы в уровне пола главного зала реактора и помещения, предназначенного для спинэхо спектрометра. Угол наклона нейтроновода в вертикальной плоскости составит $27 \mathrm{mrad}$.

Плотность нейтронного потока $d \Phi / d \lambda$ как функция длины волны, рассчитанная на выходе нейтроновода $\mathrm{CN} 1$ длиной $7500 \mathrm{~mm}$ и сечением $30 \times 200 \mathrm{~mm}^{2}$ представлена на рис. $8, a$. Узкий пик в угловом распределении нейтронов в горизонтальной плоскости в диапазоне углов $\pm 10 \mathrm{mrad}$ (рис. 8,c) сформирован нейтронами с длиной волны от 20 до $30 \AA$, в то время как широкий 
Таблица 2. Сравнительная таблица характеристик источников УХН

\begin{tabular}{l|c|c|c}
\hline \multicolumn{1}{|c|}{ Характеристика/источник } & ВВР-М & ПИК & ИЛЛ \\
\hline Поток тепловых нейтронов в источнике, $\mathrm{n} \cdot \mathrm{cm}^{-2} \mathrm{~s}^{-1}$ & $3.2 \cdot 10^{12}$ & $2.5 \cdot 10^{14}$ & $2.5 \cdot 10^{14}$ \\
Полная производительность УХН, $\mathrm{n} / \mathrm{s}$ & $1 \cdot 10^{8}$ & Не планируется & $1.2 \cdot 10^{6}$ \\
Плотность УХН в ЭДМ спектрометре $\rho_{\mathrm{EDM}}, \mathrm{cm}^{-3}$ & $1.3 \cdot 10^{4}$ & Не планируется & 10 \\
Холодные нейтроны $(2-10 \AA), \mathrm{n} /\left(\mathrm{cm}^{2} \cdot \mathrm{s}\right)^{-1}$ & $1.1 \cdot 10^{8}$ & $5.44 \cdot 10^{9}$ & $5.5 \cdot 10^{9}$ \\
Очень холодные нейтроны $(50-100 \AA), \mathrm{n} /\left(\mathrm{cm}^{2} \cdot \mathrm{s}\right)^{-1}$ & $1.2 \cdot 10^{6}$ & Не планируется & $4 \cdot 10^{6}$
\end{tabular}

пик в диапазоне углов $\pm 70 \mathrm{mrad}$ (рис. $8, c$ ) сформирован нейтронам с длиной волны от 30 до $120 \AA$. На рисунке также представлено угловое распределение нейтронов в вертикальной плоскости (рис. $8, b$ ).

\section{2. Нейтроновод $\mathrm{CN} 2$}

Нейтроновод CN2 представляет собой прямой нейтроновод сечением $30 \times 200 \mathrm{~mm}^{2}$ для вывода холодных нейтронов. Как и в случае с нейтроноводом CN1, нейтроновод CN2 необходимо поднять на $200 \mathrm{~mm}$ изза разницы в уровне полов. Плотность нейтронного потока $d \Phi / d \lambda$ как функция длины волны, рассчитанная на выходе прямого нейтроновода ХН2, приподнятого в конечной точке на $200 \mathrm{~mm}$, длиной $7500 \mathrm{~mm}$ и сечением $30 \times 200 \mathrm{~mm}^{2}$, представлена на рис. 9, $а$. Угловое распределение нейтронов в вертикальной и горизонтальной плоскостях представлены на рис. $9, b$ и $c$ соответственно.

\section{3. Нейтроновод CN3}

Пучок CN3 отклоняется от первоначального пучка $\mathrm{XH}$ на $16^{\circ}$ четырьмя секциями длиной $400 \mathrm{~mm}$ по аналогии с нейтроноводом CN1. Это даст пространство для установки научной станции на холодных нейтронах в главном зале реактора ВВР-М. Плотность нейтронного потока $d \Phi / d \lambda$ как функция длины волны, рассчитанная на выходе нейтроновода $\mathrm{CN} 3$ длиной $7500 \mathrm{~mm}$ и сечением $30 \times 200 \mathrm{~mm}^{2}$ представлена на рис. 10,a. Узкий пик в угловом распределении нейтронов в горизонтальной плоскости в диапазоне углов $\pm 10 \mathrm{mrad}$ (рис. 10,c) сформирован нейтронами с длиной волны от 20 до $30 \AA \AA$, в то время как широкий пик в диапазоне углов $\pm 70 \mathrm{mrad}$ (рис. 10,c) сформирован нейтронам с длиной волны от 30 до $120 \AA ̊$. На рисунке также представлено угловое распределение нейтронов в вертикальной плоскости (рис. 10, $b$ ).

Параметры нейтронно-оптических элементов нейтроноводов ХН и ОХН приведены в табл. 1 .

\section{Заключение}

Сравнительная таблица характеристик источников УХН приведена в табл. 2.

Таким образом, на базе действующего исследовательского реактора ВВР-М в НИЦ „Курчатовский инсти- тут“ - ПИЯФ будет создан самый высокоинтенсивный источник ультрахолодных нейтронов для научных исследований в области фундаментальной и прикладной физики. Метод получения УХН будет основан на эффекте их производства в сверхтекучем гелии изза особенностей этой квантовой жидкости. Реализация проекта со сверхтекучим гелием на реакторе ВВР-М позволит сделать источник ультрахолодных нейтронов с интенсивностью на 2 порядка больше, чем на источнике УХН в международном нейтронном центре на реакторе ИЛЛ в Гренобле. Кроме того, планируется создание пучков холодных и очень холодных нейтронов для установок по физики твердого тела, работающих в низкофоновых условиях в отдельных помещениях за пределами главного зала реактора ВВР-М.

Исследование выполнено в НИЦ „Курчатовский институт“ - ПИЯФ за счет гранта Российского научного фонда (проект № 14-22-00105).

\section{Список литературы}

[1] Serebrov A.P. // Crystallography Rep. 2011. Vol. 56. N 7. P. $1230-1237$.

[2] Korobkina E., Golub R., Wehring B.W., Young A.R. // Phys. Lett. A. 2002. Vol. 301. P. 462.

[3] Серебров А.П., Лямкин В.А., Прудников Д.В. и др. // ЖТФ. 2017. Т. 87. Вып. 2. С. 301-305.

[4] Онегин М.С., Лямкин В.А., Серебров А.П. и др. // ЖТФ. 2017. Т. 87. Вып. 4. С. 612-616.

[5] Автореф. канд. дис. Моделирование экспериментов с ультрахолодными нейтронами. Фомин А.К. Гатчина, 2006. $106 \mathrm{c}$.

[6] Серебров А.П., Коломенский Е.А., Пирожкков А.Н. и др. // Письма в ЖЭТФ. 2014. Т. 99. С. 7-11.

[7] Серебров А.П., Фомин А.К., Харитонов А.Г. и др. // ЖТФ. 2013. Т. 83. Вып. 11. С. 136-141.

[8] Серебров А.П. // УФН. 2017. Т. 185. Вып. 10. С. 1179-1201. 